\title{
Completely Operator Semi-Selfdecomposable Distributions
}

\author{
Makoto MAEJIMA (Keio University), Ken-iti SATO \\ and Toshiro WATANABE (The University of Aizu)
}

\begin{abstract}
The class $L_{\infty}(b, Q)$ of completely operator semi-selfdecomposable distributions on $\mathbf{R}^{d}$ for $b$ and $Q$ is studied. Here $0<b<1$ and $Q$ is a $d \times d$ matrix whose eigenvalues have positive real parts. This is the limiting class of the decreasing sequence of classes $L_{m}(b, Q), m=-1,0,1, \cdots$, where $L_{-1}(b, Q)$ is the class of all infinitely divisible distributions on $\mathbf{R}^{d}$ and $L_{m}(b, Q)$ is defined inductively as the class of distributions $\mu$ with characteristic function $\hat{\mu}(z)$ satisfying $\hat{\mu}(z)=\hat{\mu}\left(b^{Q^{\prime}} z\right) \hat{\rho}(z)$ for some $\rho \in L_{m-1}(b, Q)$. $Q^{\prime}$ is the transpose of $Q$. Distributions in $L_{\infty}(b, Q)$ are characterized in terms of Gaussian covariance matrices and Lévy measures. The connection with the class $\operatorname{OSS}(b, Q)$ of operator semi-stable distributions on $\mathbf{R}^{d}$ for $b$ and $Q$ is established.
\end{abstract}

\section{Introduction and a main result.}

In our previous paper [MSW99], we have introduced the class of operator semi-selfdecomposable distributions and its decreasing subclasses. To explain those classes, we start with the necessary notation. $\mathcal{P}\left(\mathbf{R}^{d}\right)$ is the class of all probability distributions on $\mathbf{R}^{d}, I\left(\mathbf{R}^{d}\right)$ is the class of all infinitely divisible distributions on $\mathbf{R}^{d}, M_{+}\left(\mathbf{R}^{d}\right)$ is the class of all $d \times d$ matrices all of whose eigenvalues have positive real parts, $Q^{\prime}$ is the transpose of $Q \in M_{+}\left(\mathbf{R}^{d}\right), I$ is the identity matrix, $\hat{\mu}(z), z \in \mathbf{R}^{d}$, is the characteristic function of $\mu \in \mathcal{P}\left(\mathbf{R}^{d}\right), \mu^{* t}, t \geq 0$, is the $t$ th convolution power of $\mu \in I\left(\mathbf{R}^{d}\right), \mathcal{L}(X)$ is the law of $X,\langle$,$\rangle is the Euclidean inner product$ in $\mathbf{R}^{d}$, and $|\cdot|$ is the norm induced by $\langle$,$\rangle in \mathbf{R}^{d}$. For $b>0, b^{Q}=\sum_{n=0}^{\infty}(n !)^{-1}(\log b)^{n} Q^{n}$. Convergence of probability distributions is always weak convergence.

Let $0<b<1, Q \in M_{+}\left(\mathbf{R}^{d}\right), m$ a nonnegative integer, and $L_{-1}(b, Q)=I\left(\mathbf{R}^{d}\right)$. A distribution $\mu \in I\left(\mathbf{R}^{d}\right)$ is said to belong to the class $L_{m}(b, Q)$ if there exists $\rho \in L_{m-1}(b, Q)$ such that $\hat{\mu}(z)=\hat{\mu}\left(b^{Q^{\prime}} z\right) \hat{\rho}(z)$. Actually the classes $L_{m}(b, Q)$ have been defined in [MSW99] in a different way and it has been shown there that the definition above is a necessary and sufficient condition for the definition in [MSW99]. Define $L_{\infty}(b, Q)$ by $L_{\infty}(b, Q)=$ $\bigcap_{m \geq 0} L_{m}(b, Q)$. We have called distributions in $L_{0}(b, Q)$ operator semi-selfdecomposable in [MSW99].

On the other hand, Jurek [J83] and Sato and Yamazato [SY85] introduced and studied the classes $L_{m}(Q)$ for $m$ a nonnegative integer or $\infty$. It has been proved in [MSW99] that $L_{m}(Q)=\bigcap_{0<b<1} L_{m}(b, Q), 0 \leq m \leq \infty$. Distributions in $L_{\infty}(Q)$ are called completely operator selfdecomposable and characterized in several ways in [SY85]. For this reason, we want to call distributions in $L_{\infty}(b, Q)$ completely operator semi-selfdecomposable. In

Received September 10, 1998 
[SY85], they studied the relationship between the class $L_{\infty}(Q)$ and that of operator stable distributions. The purpose of this paper is to give characterization of distributions in $L_{\infty}(b, Q)$ and to investigate the relationship between the class $L_{\infty}(b, Q)$ and that of operator semistable distributions.

Let $Q \in M_{+}\left(\mathbf{R}^{d}\right)$. A class $H \subset I\left(\mathbf{R}^{d}\right)$ is said to be $Q$-completely closed in the strong sense if $H$ is closed under convergence, convolution, and $Q$-type equivalence, and is closed under going to the $t$-th convolution power for any $t>0$. Here $H$ is said to be closed under $Q$-type equivalence if $\mathcal{L}(X) \in H, a>0$, and $c \in \mathbf{R}^{d}$ imply $\mathcal{L}\left(a^{-Q} X+c\right) \in H$. We can easily see from the definition that $L_{m}(b, Q), 0 \leq m \leq \infty$, are $Q$-completely closed in the strong sense, because $L_{-1}(b, Q)=I\left(\mathbf{R}^{d}\right)$ is so. Furthermore let $O S S(b, Q)$ be the class of $\mu \in I\left(\mathbf{R}^{d}\right)$ such that $\hat{\mu}(z)^{a}=\hat{\mu}\left(b^{Q^{\prime}} z\right) e^{i\langle c, z\rangle}$ for some $0<a<1$ and $c \in \mathbf{R}^{d}$. Distributions in $\operatorname{OSS}(b, Q)$ are called operator semi-stable. They are studied by Jajte [J77], Krakowiak [K80], Laha and Rohatgi [LR80], Łuczak [Ł81, Ł91], and others.

One of our main theorems is the following.

THEOREM 1.1. Let $0<b<1$ and $Q \in M_{+}\left(\mathbf{R}^{d}\right)$. Then the class $L_{\infty}(b, Q)$ is the smallest $Q$-completely closed class in the strong sense containing the class $\operatorname{OSS}(b, Q)$.

This theorem is a "semi"-version of Theorem 7.3 in [SY85]. In Section 2, we state some results we need in the subsequent sections. In Section 3, we characterize Gaussian distributions in $L_{\infty}(b, Q)$, and in Section 4, we treat purely non-Gaussian distributions in $L_{\infty}(b, Q)$. The proof of Theorem 1.1 is given in Section 5. As our results are new even in case $Q=I$, we make some remarks on this case in Section 6.

\section{Preliminary results.}

The following three propositions have recently been shown in [MSW99]. Since we need them in the subsequent sections, we state them below without proofs.

For a $d \times d$ matrix $B$ we use the following notation: $B E=\{B x: x \in E\}$ for $E \subset \mathbf{R}^{d}$ and $\left(T_{B} v\right)(E)=v(\{x: B x \in E\})$ for a measure $v$ on $\mathbf{R}^{d}$. We use a mapping $\Psi_{B}$ from the class of symmetric $d \times d$ matrices into itself defined by $\Psi_{B}(A)=A-B A B^{\prime}$. Its iteration is $\Psi_{B}^{l}=\Psi_{B} \circ \Psi_{B}^{l-1}$ for $l=2,3, \cdots$ with $\Psi_{B}^{1}=\Psi_{B}$. Also let $\mathcal{B}_{0}\left(\mathbf{R}^{d}\right)$ be the class of Borel sets $E$ in $\mathbf{R}^{d}$ such that $E \subset\{|x|>\varepsilon\}$ for some $\varepsilon>0$.

In what follows, we fix $0<b<1$ and $Q \in M_{+}\left(\mathbf{R}^{d}\right)$. We use $C_{i}, i=1,2, \cdots$, for positive constants. Following (3.4.3) in [JM93], we introduce a norm $|\cdot|_{Q}$ in $\mathbf{R}^{d}$ depending on $Q$ :

$$
|x|_{Q}=\int_{0}^{1} \frac{\left|u Q_{x}\right|}{u} d u, \quad x \in \mathbf{R}^{d}
$$

Since $C_{1} u^{C_{2}}|x| \leq\left.\left|u Q_{x \mid} \leq C_{3} u^{C_{4}}\right| x|, 0<u \leq 1| x\right|_{Q$,$} is well defined. The norm |\cdot| Q$ is comparable with the Euclidean norm $|\cdot|$, and has an advantage that, for any $x \in \mathbf{R}^{d} \backslash\{0\}, t \rightarrow$ $\left|t Q_{x}\right|_{Q}(t>0)$ is strictly increasing (Proposition 3.4.3 in [JM93]). Thus $\sup _{|x|_{Q} \leq 1} \mid b^{\left.Q_{x}\right|_{Q}<}$ 
1. Define

$$
\begin{gathered}
B=b^{Q}, \\
S_{B}=\left\{x \in \mathbf{R}^{d}:|x|_{Q} \leq 1 \text { and }\left|B^{-1} x\right|_{Q}>1\right\},
\end{gathered}
$$

and $\mathcal{B}\left(S_{B}\right)$ as the class of Borel sets in $S_{B}$. It might be better to write $S_{Q, b}$ instead of $S_{B}$, because it depends on $Q$ and $b$.

We note that all our results in this paper remain true if $S_{B}$ is defined by the usual norm in place of the norm $|\cdot| Q$, provided that $|B|=\sup _{|x| \leq 1}|B x|$, the operator norm of $B$, is less than 1. We also note that, since $\left|B^{n} x\right| \rightarrow 0$ for any $x \in \mathbf{R}^{d}$ as $n \rightarrow \infty$ and since the space is finite-dimensional, there is a positive integer $n$ such that $\left|B^{n}\right|<1$. Since $B^{n}=b^{n} Q$ and $L_{m}(b, Q) \subset L_{m}(b, n Q), 0 \leq m \leq \infty$, study of distributions in $L_{m}(b, Q)$ in the case $|B|<1$ covers all cases in some sense. However, in characterization of the class $L_{m}(b, Q)$ itself, we cannot assume that $|B|<1$. This is the reason that we use the norm $|\cdot| Q$.

PROPOSITION 2.1 (Proposition 3.2 of [MSW99]). (i) If $v$ is the Lévy measure of $\mu \in I\left(\mathbf{R}^{d}\right)$, then there exist a finite measure $v_{0}$ on $S_{B}$ and a Borel measurable function $g_{n}: S_{B} \rightarrow \mathbf{R}_{+}$for each $n \in \mathbf{Z}$ satisfying the following conditions:

(a) For $E \in \mathcal{B}\left(S_{B}\right), \nu_{0}(E)=0$ if and only if $\nu\left(B^{n} E\right)=0, \forall n \in \mathbf{Z}$,

(b) $\int_{S_{B}} \nu_{0}(d x) \sum_{n \in \mathbf{Z}}\left(\left|B^{-n} x\right|_{Q}^{2} \wedge 1\right) g_{n}(x)<\infty$,

(c) $\sum_{n \in \mathbf{Z}} g_{n}(x)>0, v_{0}$-a.e.,

(d) $\nu(E)=\int_{S_{B}} \nu_{0}(d x) \sum_{n \in \mathbf{Z}} g_{n}(x) 1_{E}\left(B^{-n} x\right), \forall E \in \mathcal{B}\left(\mathbf{R}^{d}\right)$.

These $\left\{\nu_{0}, g_{n}, n \in \mathbf{Z}\right\}$ are uniquely determined in the following sense. If $\left\{\nu_{0}, g_{n}, n \in \mathbf{Z}\right\}$ and $\left\{\tilde{v}_{0}, \tilde{g}_{n}, n \in \mathbf{Z}\right\}$ satisfy the above conditions, then there exists a Borel measurable function $h(x)$ with $0<h(x)<\infty$ such that

$$
\begin{gathered}
\tilde{\nu}_{0}(d x)=h(x) \nu_{0}(d x), \\
g_{n}(x)=h(x) \tilde{g}_{n}(x), \quad v_{0} \text {-a.e., } \forall n \in \mathbf{Z} .
\end{gathered}
$$

(ii) Conversely, if $\nu_{0}$, a finite measure on $S_{B}$, and $g_{n}, n \in \mathbf{Z}$, Borel measurable functions from $S_{B}$ into $[0, \infty)$, are given, and satisfy (b) and (c), then $v$ defined by (d) is the Lévy measure of some $\mu \in I\left(\mathbf{R}^{d}\right)$ and (a) is also satisfied.

We call $\left\{v_{0}, g_{n}, n \in \mathbf{Z}\right\}$ determined uniquely from $v$ in (i) above the $S_{B}$-representation of $\nu$. We may write $g(n, x)$ for $g_{n}(x)$ below. For $\{k(n), n \in \mathbf{Z}\}$, define $\Delta k(n)=k(n+1)-k(n)$ and $\left(\Delta^{l} k\right)(n)=\Delta\left(\Delta^{l-1} k\right)(n), l=2,3, \cdots$. The sequence $\{k(n), n \in \mathbf{Z}\}$ is called completely monotone if

$$
(-1)^{l}\left(\Delta^{l} k\right)(n) \geq 0 \quad \text { for } \forall l \geq 0, \quad \forall n \in \mathbf{Z},
$$

with $\left(\Delta^{0} k\right)(n)=k(n)$.

PROPOSITION 2.2 (Lemma 4.3 and Corollary 4.1 of [MSW99]). (i) $\quad$ If $\{k(n), n \in \mathbf{Z}\}$ is completely monotone, then

(a) there exists a unique measure $\rho$ on $(0,1]$ such that

$$
k(n)=\int_{(0,1]} x^{n} \rho(d x), \quad n \in \mathbf{Z},
$$


(b) for each $b \in(0,1)$, there exists a unique measure $\Gamma$ on $[0, \infty)$ such that

$$
k(n)=\int_{[0, \infty)} b^{n \alpha} \Gamma(d \alpha) .
$$

(ii) Conversely, $\{k(n), n \in \mathbf{Z}\}$ having the representation (2.1) is completely monotone.

Proposition 2.3 (Theorem 3.1 of [MSW99]). Let $0 \leq m \leq \infty, \mu \in I\left(\mathbf{R}^{d}\right), A$ its Gaussian covariance matrix, $v$ its Lévy measure, and let $\left\{\nu_{0}, g(n, x), n \in \mathbf{Z}\right\}$ be the $S_{B^{-}}$ representation of $\nu$. Then the following three statements are equivalent:

(i) $\mu \in L_{m}(b, Q)$.

(ii) $\Psi_{B}^{l}(A), 1 \leq l \leq m+1$, are nonnegative definite, and $\left(I-T_{B}\right)^{l} v \geq 0,1 \leq l \leq$ $m+1$, on $\mathcal{B}_{0}\left(\mathbf{R}^{d}\right)$.

(iii) $\Psi_{B}^{l}(A), 1 \leq l \leq m+1$, are nonnegative definite, and $(-1)^{l}\left(\Delta^{l} g\right)(n, x) \geq 0$, $n \in \mathbf{Z}$, v $v_{0}$-a.e.x for $1 \leq l \leq m+1$.

(In the above, when $m=\infty, 1 \leq l \leq m+1$ should be read as $1 \leq l<\infty$.)

\section{Gaussian distributions in $L_{\infty}(b, Q)$.}

The following are generalizations of some results in [SY85] to "semi"-version.

Let $\left\{\beta_{1}, \cdots, \beta_{p}\right\}$ be the distinct eigenvalues of $B=b^{Q}$, and let $f(\zeta)$ be the minimal polynomial of $B$. Decompose it into linear factors

$$
f(\zeta)=\left(\zeta-\beta_{1}\right)^{n_{1}} \cdots\left(\zeta-\beta_{p}\right)^{n_{p}}
$$

where, for $1 \leq j \leq p, n_{j}$ is a positive integer not exceeding the multiplicity of $\beta_{j}$. Let

$$
V_{j}=\operatorname{Ker}\left(B-\beta_{j} I\right)^{n_{j}} \quad \text { in } \mathbf{C}^{d}(1 \leq j \leq p) .
$$

Then

$$
\mathbf{C}^{d}=V_{1} \oplus \cdots \oplus V_{p}
$$

Let $T_{j}$ be the projector of $\mathbf{C}^{d}$ onto $V_{j}$ in this direct sum decomposition. Similarly we denote

$$
V_{j}^{\prime}=\operatorname{Ker}\left(B^{\prime}-\bar{\beta}_{j} I\right)^{n_{j}} \quad \text { in } \mathbf{C}^{d}(1 \leq j \leq p),
$$

and obtain

$$
\mathbf{C}^{d}=V_{1}^{\prime} \oplus \cdots \oplus V_{p}^{\prime}
$$

Then the projector of $\mathbf{C}^{d}$ onto $V_{j}^{\prime}$ in this direct sum decomposition coincides with the adjoint operator $T_{j}^{\prime}$ of $T_{j}$. For $j \neq k, V_{j}^{\prime}$ and $V_{k}$ are orthogonal, where we use the Hermitian inner product denoted also by $\langle$,$\rangle . The following is a characterization of Gaussian distribution in$ $L_{\infty}(b, Q)$.

THEOREM 3.1. Let $\mu$ be a Gaussian distribution with covariance matrix A. Then the following three statements are equivalent:

(i) $\mu \in L_{\infty}(b, Q)$.

(ii) $\left(B-\beta_{j}\right) A T_{j}^{\prime}=0$, for $1 \leq j \leq p$.

(iii) (a) $A\left(B^{\prime}-\bar{\beta}_{j}\right) T_{j}^{\prime}=0$, for $1 \leq j \leq p$, and (b) $T_{k} A T_{j}^{\prime}=0$ for $j \neq k$. 
To prove the theorem, we need a lemma.

LEMMA 3.1. Let $\mu$ be a Gaussian distribution with covariance matrix A. Then $\mu \in$ $L_{\infty}(b, Q)$ if and only if for any $z \in \mathbf{R}^{d},\left\langle A B^{\prime n} z, B^{\prime n} z\right\rangle, n \in \mathbf{Z}$, is completely monotone.

PRoOF. Set $k_{z}(n)=\left\langle A B^{\prime n} z, B^{\prime n} z\right\rangle, n \in \mathbf{Z}, z \in \mathbf{R}^{d}$. Then observe that, for each $l \geq 1$, $(-1)^{l}\left(\Delta^{l} k_{z}\right)(n) \geq 0, \forall n \in \mathbf{Z}, \forall z \in \mathbf{R}^{d}$, if and only if $\Psi_{B}^{l}(A)$ is nonnegative definite. The nonnegative definiteness of $\Psi_{B}^{l}(A)$ for all $l \geq 0$ is a necessary and sufficient condition for that the Gaussian $\mu$ is in $L_{\infty}(b, Q)$ by Proposition 2.3. This concludes the lemma.

Proof of THEOREM 3.1. We first show (i) $\Rightarrow$ (iii). To show (iii)(a), it is enough to prove that, for any integer $k \geq 1$ and $z_{0} \in \mathbf{C}^{d}$,

$$
\left(B^{\prime}-\bar{\beta}_{j}\right)^{k} z_{0}=0 \text { implies } A\left(B^{\prime}-\bar{\beta}_{j}\right) z_{0}=0 .
$$

We prove this by induction in $k$. If $k=1$, the assertion is trivial. Suppose that (3.1) is true for $k-1$ in place of $k$, and assume $\left(B^{\prime}-\bar{\beta}_{j}\right)^{k} z_{0}=0$. Since $B^{\prime n}\left(B^{\prime}-\bar{\beta}_{j}\right)=\left(B^{\prime}-\bar{\beta}_{j}\right) B^{\prime n}$ for any $n \in \mathbf{Z}$, we have $\left(B^{\prime}-\bar{\beta}_{j}\right)^{k-2+l} B^{\prime n} z_{0}=0$ for any $l \geq 2$ and $n \in \mathbf{Z}$. Hence by the induction hypothesis,

$$
A\left(B^{\prime}-\bar{\beta}_{j}\right)^{l} B^{\prime n} z_{0}=0 \text { for } l \geq 2 \text { and } n \in \mathbf{Z} .
$$

Let

$$
L(n)=\left\langle A B^{\prime n} z_{0}, B^{\prime n} z_{0}\right\rangle \quad \text { for } n \in \mathbf{Z} .
$$

We claim that

$$
L(n)=\left|\beta_{j}\right|^{2 n}\left\{\left\langle A z_{0}, z_{0}\right\rangle+2 n \Re\left\langle A z_{0}, z_{1}\right\rangle+n^{2}\left\langle A z_{1}, z_{1}\right\rangle\right\}, \quad n \in \mathbf{Z},
$$

where $z_{1}=\bar{\beta}_{j}^{-1}\left(B^{\prime}-\bar{\beta}_{j}\right) z_{0}$. If $n=0$, this is trivial. We write $z_{l}=\bar{\beta}_{j}^{-l}\left(B^{\prime}-\bar{\beta}_{j}\right)^{l} z_{0}$. If $n \geq 1$, then

$$
B^{\prime n} z_{0}=\left(\bar{\beta}_{j}+\left(B^{\prime}-\bar{\beta}_{j}\right)\right)^{n} z_{0}=\bar{\beta}_{j}^{n} \sum_{l=0}^{n}\left(\begin{array}{l}
n \\
l
\end{array}\right) z_{l}
$$

and, by (3.2),

$$
L(n)=\left\langle\bar{\beta}_{j}^{n} A\left(z_{0}+n z_{1}\right), B^{\prime n} z_{0}\right\rangle=\left\langle\bar{\beta}_{j}^{n}\left(z_{0}+n z_{1}\right), \bar{\beta}_{j}^{n} A\left(z_{0}+n z_{1}\right)\right\rangle,
$$

which is (3.3). Suppose $n \leq-1$ and write $n=-h$. Let $w=B^{\prime n} z_{0}-\bar{\beta}_{j}^{n}\left(z_{0}+n z_{1}\right)$. Then

$$
\begin{aligned}
w & =B^{\prime n}\left\{z_{0}-\bar{\beta}_{j}^{n} B^{\prime h}\left(z_{0}+n z_{1}\right)\right\} \\
& =B^{\prime n}\left\{z_{0}-\sum_{l=0}^{h}\left(\begin{array}{l}
h \\
l
\end{array}\right)\left(z_{l}+n z_{l+1}\right)\right\} \\
& =B^{\prime n}\left\{n^{2} z_{2}-\sum_{l=2}^{h}\left(\begin{array}{l}
h \\
l
\end{array}\right)\left(z_{l}+n z_{l+1}\right)\right\},
\end{aligned}
$$


where the sum over $2 \leq l \leq h$ is considered as zero if $h=1$. Hence by (3.2), $A w=0$. Thus

$$
\begin{aligned}
L(n) & =\left\langle A B^{\prime n} z_{0}-A w, B^{\prime n} z_{0}\right\rangle \\
& =\left\langle\bar{\beta}_{j}^{n} A\left(z_{0}+n z_{1}\right), B^{\prime n} z_{0}\right\rangle \\
& =\left\langle\bar{\beta}_{j}^{n}\left(z_{0}+n z_{1}\right), A B^{\prime n} z_{0}-A w\right\rangle \\
& =\left\langle\bar{\beta}_{j}^{n}\left(z_{0}+n z_{1}\right), \bar{\beta}_{j}^{n} A\left(z_{0}+n z_{1}\right)\right\rangle .
\end{aligned}
$$

Hence (3.3) is true for all $n \in \mathbf{Z}$. Since $L(n)$ is completely monotone in $n$ by Lemma 3.1 under the assumption that $\mu \in L_{\infty}(b, Q)$, we have

$$
L(n)=\int_{(0,1]} \beta^{n} \rho(d \beta)
$$

for some measure $\rho$ by Proposition 2.2. If we let

$$
\begin{aligned}
& E_{1}=\left\{\beta \in(0,1]: \beta>\left|\beta_{j}\right|^{2}\right\}, \\
& E_{2}=\left\{\beta \in(0,1]: \beta<\left|\beta_{j}\right|^{2}\right\},
\end{aligned}
$$

then from (3.3) and (3.4)

$$
\begin{aligned}
\rho\left(\left\{\left|\beta_{j}\right|^{2}\right\}\right)+\int_{E_{1}}\left(\frac{\beta}{\left|\beta_{j}\right|^{2}}\right)^{n} \rho(d \beta)+\int_{E_{2}}\left(\frac{\beta}{\left|\beta_{j}\right|^{2}}\right)^{n} \rho(d \beta) \\
=\left\langle A z_{0}, z_{0}\right\rangle+2 n \Re\left\langle A z_{0}, z_{1}\right\rangle+n^{2}\left\langle A z_{1}, z_{1}\right\rangle=I,
\end{aligned}
$$

say. If $\rho\left(E_{1}\right)>0$, then there exists $\varepsilon>0$ such that $\rho\left((1+\varepsilon)\left|\beta_{j}\right|^{2} \leq \beta \leq 1\right)>0$, and hence

$$
I \geq(1+\varepsilon)^{n} \rho\left((1+\varepsilon)\left|\beta_{j}\right|^{2} \leq \beta \leq 1\right), \quad n>0 .
$$

Letting $n \rightarrow \infty$, we get a contradiction. Thus $\rho\left(E_{1}\right)=0$. Similarly, if $\rho\left(E_{2}\right)>0$, then there exists $\varepsilon>0$ such that

$$
I \geq(1-\varepsilon)^{n} \rho\left(0<\beta \leq(1-\varepsilon)\left|\beta_{j}\right|^{2}\right), \quad n<0,
$$

and letting $n \rightarrow-\infty$ yields a contradiction. Thus $\rho\left(E_{2}\right)=0$. Consequently,

$$
\int_{(0,1]} \beta^{n} \rho(d \beta)=\left|\beta_{j}\right|^{2 n} \rho\left(\left\{\left|\beta_{j}\right|^{2}\right\}\right)=\left|\beta_{j}\right|^{2 n}\left\langle A z_{0}, z_{0}\right\rangle,
$$

and $\left\langle A z_{1}, z_{1}\right\rangle=0$. By Lemma 3.1 of [SY85], we conclude that $A z_{1}=0$. This proves (3.1).

Let us show (iii)(b). It is enough to show that

$$
\left\langle A z_{0}, w_{0}\right\rangle=0 \text { for any } z_{0} \in V_{j}^{\prime} \text { and } w_{0} \in V_{k}^{\prime} \text { with } j \neq k .
$$

Since $V_{j}^{\prime}$ and $V_{k}^{\prime}$ are invariant under $B^{\prime h}, h \in \mathbf{Z}$, we have, by (iii)(a),

$$
A\left(B^{\prime}-\bar{\beta}_{j}\right)^{l} B^{\prime h} z_{0}=A\left(B^{\prime}-\bar{\beta}_{k}\right)^{l} B^{\prime h} w_{0}=0 \quad \text { for } l \geq 1 \text { and } h \in \mathbf{Z} \text {. }
$$

Hence, for $n \in \mathbf{Z}$,

$$
\begin{aligned}
L_{ \pm}(n) & \left\langle A B^{\prime n}\left(z_{0} \pm w_{0}\right), B^{\prime n}\left(z_{0} \pm w_{0}\right)\right\rangle \\
& =\left|\beta_{j}\right|^{2 n}\left\langle A z_{0}, z_{0}\right\rangle \pm 2 \Re \bar{\beta}_{j}^{n} \beta_{k}^{n}\left\langle A z_{0}, w_{0}\right\rangle+\left|\beta_{k}\right|^{2 n}\left\langle A w_{0}, w_{0}\right\rangle .
\end{aligned}
$$


We consider two cases.

Case I $\left(\left|\beta_{j}\right| \neq\left|\beta_{k}\right|\right)$. As before, there exist measures $\rho_{+}$and $\rho_{-}$on $(0,1]$ such that

$$
L_{ \pm}(n)=\int_{(0,1]} \beta^{n} \rho_{ \pm}(d \beta)
$$

Let us show

$$
\begin{gathered}
\rho_{ \pm}\left(\left\{\left|\beta_{j}\right|^{2}\right\}\right)=\left\langle A z_{0}, z_{0}\right\rangle \\
\rho_{ \pm}\left(\left\{\left|\beta_{k}\right|^{2}\right\}\right)=\left\langle A w_{0}, w_{0}\right\rangle .
\end{gathered}
$$

Without loss of generality, we assume that $\left|\beta_{j}\right|<\left|\beta_{k}\right|$. As in the case of $L(n)$, we observe

$$
\rho_{ \pm}\left(\beta<\left|\beta_{j}\right|^{2}\right)=\rho_{ \pm}\left(\beta>\left|\beta_{k}\right|^{2}\right)=0 .
$$

It follows from (3.5) and (3.6) that

$$
\begin{aligned}
\left\langle A w_{0}, w_{0}\right\rangle & =\lim _{n \rightarrow \infty} \frac{L_{ \pm}(n)}{\left|\beta_{k}\right|^{2 n}} \\
& =\lim _{n \rightarrow \infty} \int_{\beta<\left|\beta_{k}\right|^{2}}\left(\frac{\beta}{\left|\beta_{k}\right|^{2}}\right)^{n} \rho_{ \pm}(d \beta)+\rho_{ \pm}\left(\left\{\left|\beta_{k}\right|^{2}\right\}\right) \\
& =\rho_{ \pm}\left(\left\{\left|\beta_{k}\right|^{2}\right\}\right) .
\end{aligned}
$$

This shows (3.8). (3.7) can be shown similarly, by considering $L_{ \pm}(n) /\left|\beta_{j}\right|^{2 n}$ and letting $n \rightarrow-\infty$. Thus we have

$$
\pm 2 \Re \bar{\beta}_{j}^{n} \beta_{k}^{n}\left\langle A z_{0}, w_{0}\right\rangle=\int_{\left(\left|\beta_{j}\right|^{2},\left|\beta_{k}\right|^{2}\right)} \beta^{n} \rho_{ \pm}(d \beta) \geq 0,
$$

concluding $\Re\left\langle A z_{0}, w_{0}\right\rangle=0$. Since $i z_{0} \in V_{j}$, we also have $\Im\left\langle A z_{0}, w_{0}\right\rangle=0$.

Case II $\left(\left|\beta_{j}\right|=\left|\beta_{k}\right|\right)$. We have

$$
L_{+}(n)=\left|\beta_{j}\right|^{2 n}\left\{\left\langle A z_{0}, z_{0}\right\rangle+\left\langle A w_{0}, w_{0}\right\rangle+2 \Re\left(\frac{\beta_{k}}{\beta_{j}}\right)^{n}\left\langle A z_{0}, w_{0}\right\rangle\right\} .
$$

As in the case of $L(n)$, we see that

$$
L_{+}(n)=\int_{(0,1]} \beta^{n} \rho_{+}(d \beta)=\text { const. } \times\left|\beta_{j}\right|^{2 n} .
$$

Therefore, $\Re\left(\beta_{k} / \beta_{j}\right)^{n}\left\langle A z_{0}, w_{0}\right\rangle$ is independent of $n$. Since $\beta_{k} / \beta_{j} \neq 1$, we have $\left\langle A z_{0}, w_{0}\right\rangle=$ 0 . This conclude (iii)(b).

We next show (iii) $\Rightarrow$ (i). By the Jordan decomposition of $B^{\prime}$, we can find a basis $\left\{z_{j l k}=\right.$ $\left.\bar{\beta}_{j}^{-k}\left(B^{\prime}-\bar{\beta}_{j}\right)^{k} z_{j l}, 1 \leq j \leq p, 1 \leq l \leq l_{j}, 0 \leq k \leq k(j, l)\right\}$ of $\mathbf{C}^{d}$ for some integers $l_{j}$ and $k(j, l)$. Here $z_{j l} \in V_{j}^{\prime}\left(1 \leq l \leq l_{j}\right)$ and $\left(B^{\prime}-\bar{\beta}_{j}\right)^{k(j, l)+1} z_{j l}=0$. Thus, for any $z \in \mathbf{C}^{d}$,

$$
z=\sum_{j, l, k} c_{j l k} z_{j l k} \quad \text { with some } c_{j l k} \in \mathbf{C}
$$


and, hence,

$$
B^{\prime n} z=\sum_{j, l, k} \bar{\beta}_{j}^{n} c_{j l k} \sum_{m=0}^{(k(j, l)-k) \wedge n}\left(\begin{array}{c}
n \\
m
\end{array}\right) z_{j, l, k+m}
$$

for all $n \in \mathbf{Z}_{+}$. Therefore, by (iii) for any $n \in \mathbf{Z}$,

$$
A\left(B^{\prime n} z-\sum_{j, l} \bar{\beta}_{j}^{n} c_{j l 0} z_{j l 0}\right)=0 .
$$

Hence, for $n \in \mathbf{Z}$,

$$
\begin{aligned}
\left\langle A B^{\prime n} z, B^{\prime n} z\right\rangle & =\sum_{j=1}^{p}\left|\beta_{j}\right|^{2 n}\left\langle A \sum_{l} c_{j l 0} z_{j l 0}, \sum_{l} c_{j l 0} z_{j l 0}\right\rangle \\
& =\int_{(0,1]} \beta^{n} \rho(d \beta),
\end{aligned}
$$

which is completely monotone, if we define $\rho$ by $\rho\left(\left\{\left|\beta_{j}\right|^{2}\right\}\right)=\left\langle A \sum_{l} c_{j l 0} z_{j l 0}, \sum_{l} c_{j l 0} z_{j l 0}\right\rangle$ and $\rho\left((0,1] \backslash\left\{\left|\beta_{1}\right|^{2}, \cdots,\left|\beta_{p}\right|^{2}\right\}\right)=0$. It follows from Lemma 3.1 that $\mu \in L_{\infty}(b, Q)$.

We finally show (ii) $\Rightarrow$ (iii). (iii) $\Rightarrow$ (ii) is easily seen, because we have, for any $z, w \in \mathbf{C}^{d}$,

$$
\left\langle\left(B-\beta_{j}\right) A T_{j}^{\prime} z, w\right\rangle=\sum_{k=1}^{p}\left\langle T_{j}^{\prime} z, A\left(B^{\prime}-\bar{\beta}_{j}\right) T_{k}^{\prime} w\right\rangle=0,
$$

using (iii)(a) for $k=j$ and (iii)(b) for $k \neq j$. As to (ii) $\Rightarrow$ (iii), we have

$$
\left\langle A\left(B^{\prime}-\bar{\beta}_{j}\right) T_{j}^{\prime} z,\left(B^{\prime}-\bar{\beta}_{j}\right) T_{j}^{\prime} z\right\rangle=\left\langle\left(B-\beta_{j}\right) A\left(B^{\prime}-\bar{\beta}_{j}\right) T_{j}^{\prime} z, T_{j}^{\prime} z\right\rangle=0,
$$

which together with Lemma 3.1 of [SY85] implies that $A\left(B^{\prime}-\bar{\beta}_{j}\right) T_{j}^{\prime} z=0$, namely (iii)(a). Also we have (iii)(b), since (ii) implies that $A T_{j}^{\prime}$ has its range in $V_{j}$. The proof of Theorem 3.1 is thus complete.

Theorem 3.1 uses a direct sum decomposition of $\mathbf{C}^{d}$. Let us consider the corresponding decomposition of $\mathbf{R}^{d}$, and then prove a decomposition theorem of Gaussian distributions in $L_{\infty}(b, Q)$. For this purpose we arrange the distinct eigenvalues of $B=b^{Q}$ in such a way that $\beta_{1}, \cdots, \beta_{q}$ are real and $\beta_{q+1}, \cdots, \beta_{p}$ are not real, $\beta_{j}=\bar{\beta}_{j+r}(q+1 \leq j \leq q+r)$, and $q+2 r=p$. Here $q$ or $r$ may possibly be zero. Let $\gamma_{j}$ and $\delta_{j}$ be the real and the imaginary part of $\beta_{j}$, respectively. The real factorization of the minimal polynomial $f(\zeta)$ of $B^{\prime}$ is

$$
f(\zeta)=f_{1}(\zeta)^{n_{1}} \cdots f_{q+r}(\zeta)^{n_{q+r}}
$$

where $f_{j}(\zeta)=\zeta-\beta_{j}=\zeta-\gamma_{j}, 1 \leq j \leq q$, and $f_{j}(\zeta)=\left(\zeta-\gamma_{j}\right)^{2}+\delta_{j}^{2}, q+1 \leq j \leq q+r$. Let

$$
W_{j}^{\prime}=\operatorname{Ker} f_{j}\left(B^{\prime}\right)^{n_{j}} \quad \text { in } \mathbf{R}^{d}, \quad 1 \leq j \leq q+r
$$

Then

$$
\mathbf{R}^{d}=W_{1}^{\prime} \oplus \cdots \oplus W_{q+r}^{\prime}
$$


As in the proof $(\mathrm{iii}) \Rightarrow(\mathrm{i})$ of Theorem 3.1 , let

$$
\left\{z_{j l k}=\bar{\beta}_{j}^{-k}\left(B^{\prime}-\bar{\beta}_{j}\right)^{k} z_{j l}: 1 \leq j \leq p, 1 \leq l \leq l_{j}, 0 \leq k \leq k(j, l)\right\}
$$

be a basis of $\mathbf{C}^{d}$, where $z_{j l} \in V_{j}^{\prime}$ and $\left(B^{\prime}-\bar{\beta}_{j}\right)^{k(j, l)+1} z_{j l}=0$. For $1 \leq j \leq q$, we can choose $z_{j l}$ real so that $\left\{z_{j l k}: 1 \leq l \leq l_{j}, 0 \leq k \leq k(j, l)\right\}$ is a basis of $W_{j}^{\prime}$. For $q+1 \leq j \leq q+r$, we have $l_{j}=l_{j+r}$ and $k(j, l)=k(j+r, l)$ and we can choose $z_{j l}$ and $z_{j+r, l}$ in such a way that $z_{j l}=\bar{z}_{j+r, l}$. Let $\xi_{j l k}$ and $\eta_{j l k}$ be the real and the imaginary part of $z_{j l k}$, respectively, for $q+1 \leq j \leq q+r$. Here complex conjugates, real parts, and imaginary parts of vectors in $\mathbf{C}^{d}$ are taken component-wise. The system $\left\{\xi_{j l k}, \eta_{j l k}: 1 \leq l \leq l_{j}, 0 \leq k \leq k(j, l)\right\}$ is then a basis of $W_{j}^{\prime}$. The following theorem gives a matrix representation when these bases are used.

THEOREM 3.2. Let $\mu$ be Gaussian with covariance matrix $A$. Then $\mu \in L_{\infty}(b, Q)$ if and only if the following four conditions are satisfied:

(i) $\left\langle A z_{j l k}, z_{j l k}\right\rangle=0$ for $1 \leq j \leq q, 1 \leq l \leq l_{j}, k \geq 1$,

(ii) $\left\langle A \xi_{j l k}, \xi_{j l k}\right\rangle=\left\langle A \eta_{j l k}, \eta_{j l k}\right\rangle=0$ for $q+1 \leq j \leq q+r, 1 \leq l \leq l_{j}, k \geq 1$,

(iii) $\left\langle A \xi_{j l 0}, \xi_{j m 0}\right\rangle=\left\langle A \eta_{j l 0}, \eta_{j m 0}\right\rangle$ and $\left\langle A \xi_{j l 0}, \eta_{j m 0}\right\rangle=-\left\langle A \eta_{j l 0}, \xi_{j m 0}\right\rangle$ for $q+1 \leq$ $j \leq q+r, 1 \leq l \leq l_{j}, 1 \leq m \leq l_{j}$ with $l=m$ inclusive,

(iv) $\langle A z, w\rangle=0$ for $z \in W_{j}^{\prime}, w \in W_{k}^{\prime}$, for $1 \leq j \leq q+r, 1 \leq k \leq q+r$ with $j \neq k$.

PROOF. The proof is exactly the same as that of Theorem 4.1 of [SY85]. So we omit it here.

Let us consider the direct sum decomposition of $\mathbf{R}^{d}$ associated with $B=b^{Q}$. Let

$$
W_{j}=\operatorname{Ker} f_{j}(B)^{n_{j}} \quad \text { in } \mathbf{R}^{d}, \quad 1 \leq j \leq q+r .
$$

Then

$$
\mathbf{R}^{d}=W_{1} \oplus \cdots \oplus W_{q+r} .
$$

This is the decomposition dual to (3.10). Let $U_{j}$ be the projector of $\mathbf{R}^{d}$ onto $W_{j}$ in the decomposition (3.11). The transposed matrix $U_{j}^{\prime}$ of $U_{j}$ is the projector onto $W_{j}^{\prime}$ in the decomposition (3.10). For $q+1 \leq j \leq q+r$, we have $V_{j}=\bar{V}_{j+r}$ and $T_{j} x=\overline{T_{j+r} x}$ for $x \in \mathbf{R}^{d}$. Thus $U_{j} x=T_{j} x+T_{j+r} x$ for $x \in \mathbf{R}^{d}$ for $q+1 \leq j \leq q+r$. For $1 \leq j \leq q$, we have $U_{j} x=T_{j} x$ for $x \in \mathbf{R}^{d}$. Let

$$
N_{j}=\operatorname{Ker} f_{j}(B) \quad \text { in } \mathbf{R}^{d}, \quad 1 \leq j \leq q+r .
$$

THEOREM 3.3. Suppose that $\mu$ is a centered Gaussian distribution in $L_{\infty}(b, Q)$. Then, the support of $\mu$ is a B-invariant linear subspace of $\mathbf{R}^{d}$ and the minimal polynomial of the restriction of $B$ to the support of $\mu$ does not have double roots. There exists a unique decomposition $\mu=\mu_{1} * \cdots * \mu_{q+r}$, where each $\mu_{j}$ is a centered Gaussian distribution such that $\mu_{j} \in L_{\infty}(b, Q)$ and the support of $\mu_{j}$ is contained $N_{j}$ and hence in $W_{j}$.

PROOF. Again, the proof is exactly the same as that of Theorem 4.2 of [SY85]. So we omit it here. 
REMARK 3.1. The $\mu_{j}$ in Theorem 3.3 is centered Gaussian with covariance matrix $A_{j}=U_{j} A U_{j}^{\prime}$. Thus, since $\mu_{j} \in L_{\infty}(b, Q)$, by Theorem 3.1,

$$
\begin{aligned}
\hat{\mu}_{j}\left(B^{\prime} z\right) & =\exp \left\{-\frac{1}{2}\left\langle A_{j} B^{\prime} z, B^{\prime} z\right\rangle\right\} \\
& =\exp \left\{-\frac{1}{2}\left\langle A_{j} B^{\prime} U_{j}^{\prime} z, B^{\prime} U_{j}^{\prime} z\right\rangle\right\} \\
& =\exp \left\{-\frac{1}{2}\left|\beta_{j}\right|^{2}\left\langle A_{j} U_{j}^{\prime} z, U_{j}^{\prime} z\right\rangle\right\} \\
& =\exp \left\{-\frac{1}{2}\left|\beta_{j}\right|^{2}\left\langle A_{j} z, z\right\rangle\right\} \\
& =\hat{\mu}_{j}(z)^{\left|\beta_{j}\right|^{2}},
\end{aligned}
$$

which means that $\mu_{j} \in \operatorname{OSS}(b, Q)$.

Combining Theorem 3.3 and Remark 3.1, we have

THEOREM 3.4. Let $s$ be the number of distinct absolute values of eigenvalues of $B=$ $b^{Q}$. If $\mu$ is a Gaussian distribution in $L_{\infty}(b, Q)$, then $\mu$ can be expressed as the convolution of at most $s$ Gaussian distributions in $\operatorname{OSS}(b, Q)$.

EXAMPLE. For $d=2,3,4$, explicit forms of the covariance matrices of Gaussian distributions in $L_{\infty}(Q)$ are given in [SY85]. Let $d=2$ and let $I_{G}$ be the class of all Gaussian distributions on $\mathbf{R}^{2}$. Let $\mu \in I_{G}$ with covariance matrix $A$.

First consider the case $Q=\left(\begin{array}{ll}\gamma & 1 \\ 0 & \gamma\end{array}\right)$ with $\gamma>0$. For $0<b<1, b^{Q}=b^{\gamma}\left(\begin{array}{cc}1 & \log b \\ 0 & 1\end{array}\right)$ and hence $b^{Q}$ has the Jordan form $\left(\begin{array}{cc}b^{\gamma} & 1 \\ 0 & b^{\gamma}\end{array}\right)$. As is shown in [SY85], $\mu \in L_{\infty}(Q)$ if and only if $A=\left(\begin{array}{ll}a & 0 \\ 0 & 0\end{array}\right), a \geq 0$. As our Theorem 3.2 is formally the same as Theorem 4.1 of [SY85], we see that $\mu \in L_{\infty}(b, Q)$ if and only if $A=\left(\begin{array}{ll}a & 0 \\ 0 & 0\end{array}\right), a \geq 0$. Thus $L_{\infty}(b, Q) \cap I_{G}=L_{\infty}(Q) \cap I_{G}$ for any $0<b<1$ in this case.

Next consider the case $Q=\left(\begin{array}{cc}\gamma & -\delta \\ \delta & \gamma\end{array}\right)$ with $\gamma>0$ and $\delta \in \mathbf{R} \backslash\{0\}$. Then, for $0<b<1$, $b^{Q}=b^{\gamma}\left(\begin{array}{cc}\cos \theta & -\sin \theta \\ \sin \theta & \cos \theta\end{array}\right)$ with $\theta=\delta \log b$. In this case, $\mu \in L_{\infty}(Q)$ if and only if $A=a I$, $a \geq 0$, as is shown [SY85]. If $b=e^{n \pi / \delta}$ with $n \in \mathbf{Z}$ and $n / \delta<0$, then $b^{Q}=b^{\gamma}(-1)^{n} I$ and hence $L_{\infty}(b, Q) \cap I_{G}=I_{G}$. Otherwise, $b^{Q}$ is of the same type as $Q$ and $L_{\infty}(b, Q) \cap I_{G}=$ $L_{\infty}(Q) \cap I_{G}$. Thus, $L_{\infty}(b, Q) \cap I_{G}$ truly depends on $b$ and, for some $b, L_{\infty}(b, Q) \cap I_{G}$ is strictly larger than $L_{\infty}(Q) \cap I_{G}$. 
4. Purely non-Gaussian distributions in $L_{\infty}(b, Q)$.

Now we give a representation of the Lévy measure of purely non-Gaussian $\mu \in L_{\infty}(b, Q)$. For each $x \in \mathbf{R}^{d} \backslash\{0\}$, let

$$
\begin{gathered}
\beta(x)=\max \left\{\left|\beta_{j}\right|: 1 \leq j \leq q+2 r, T_{j} x \neq 0\right\}, \\
n(x, j)=\max \left\{n: n \geq 0,\left(B-\beta_{j}\right)^{n} T_{j} x \neq 0 \text { for } T_{j} x \neq 0\right\}, \\
n(x)=\max \left\{n(x, j): 1 \leq j \leq q+2 r, T_{j} x \neq 0,\left|\beta_{j}\right|=\beta(x)\right\}, \\
\gamma(x)=\frac{\log \beta(x)}{\log b} .
\end{gathered}
$$

We show the following. Given two measurable spaces $\left(\Theta_{1}, \mathcal{B}_{1}\right)$ and $\left(\Theta_{2}, \mathcal{B}_{2}\right)$, we say that $\left\{\Gamma_{\theta_{1}}, \theta_{1} \in \Theta_{1}\right\}$, a system of measures on $\left(\Theta_{2}, \mathcal{B}_{2}\right)$, is measurable in $\theta_{1}$ if $\Gamma_{\theta_{1}}(E)$ is measurable in $\theta_{1}$ for every $E \in \mathcal{B}_{2}$.

THEOREM 4.1. (i) Suppose that $\mu$ is a purely non-Gaussian distribution in $I\left(\mathbf{R}^{d}\right)$ with nonzero Lévy measure $v$. Then $\mu \in L_{\infty}(b, Q)$ if and only if $v$ is expressed as

$$
\nu(E)=\int_{S_{B}} \nu_{0}(d x) \int_{(0,2 \gamma(x))} \Gamma_{x}(d \alpha) \sum_{n \in \mathbf{Z}} b^{n \alpha} 1_{E}\left(B^{-n} x\right), \quad E \in \mathcal{B}\left(\mathbf{R}^{d}\right),
$$

where $\nu_{0}$ is a nonzero finite measure on $S_{B}$ and $\Gamma_{x}, x \in S_{B}$, are nonzero finite measures on $(0, \infty)$ measurable in $x$, each $\Gamma_{x}$ is concentrated on $(0,2 \gamma(x))$ and

$$
\int_{S_{B}} \nu_{0}(d x) \int_{(0,2 \gamma(x))} \Gamma_{x}(d \alpha) \sum_{n \in \mathbf{Z}} b^{n \alpha}\left(\left|B^{-n} x\right|_{Q}^{2} \wedge 1\right)<\infty .
$$

(ii) If a nonzero finite measure $\nu_{0}$ on $S_{B}$ and nonzero finite measures $\Gamma_{x}, x \in S_{B}$, on $(0, \infty)$ measurable in $x$ are given and if each $\Gamma_{x}$ is concentrated on $(0,2 \gamma(x))$ and (4.2) is satisfied, then the measure $v$ defined by (4.1) is the Lévy measure of some $\mu \in I\left(\mathbf{R}^{d}\right)$.

(iii) If $\mu \in L_{\infty}(b, Q)$ has nonzero Lévy measure $v$ and if $\nu$ is expressed by $\nu_{0}$ and $\Gamma_{x}$ as in (i), then $\nu_{0}$ and $\Gamma_{x}$ are unique in the following sense: if $\tilde{v}$ and $\tilde{\Gamma}_{x}$ give another expression of $v$, then there exists a Borel measurable function $h(x)$ with $0<h(x)<\infty$ such that $\tilde{v}_{0}(d x)=h(x) \nu_{0}(d x)$ and $\Gamma(d \alpha)=h(x) \tilde{\Gamma}_{x}(d \alpha)$ for $v_{0}$-a.e. $x$. The measures $\Gamma_{x}$ necessarily satisfy

$$
\int_{(0,2 \gamma(x))}\left(\alpha^{-1}+(2 \gamma(x)-\alpha)^{-2 n(x)-1}\right) \Gamma_{x}(d \alpha)<\infty, \quad v_{0} \text {-a.e. } x .
$$

LEMMA 4.1. There exist positive constants $C_{j}(j=5,6,7)$ and $b_{j}(x)(j=1,2,3)$ such that, for $x \in S_{B}$,

$$
\begin{gathered}
\left|B^{k} x\right|_{Q} \leq C_{5} \beta(x)^{k} k^{n(x)} \quad \text { for } k \geq 1, \\
\left|B^{k} x\right|_{Q} \geq b_{2}(x) \beta(x)^{k} k^{n(x)} \quad \text { for } k \geq b_{1}(x), \\
C_{6} \alpha^{-1}+b_{3}(x)(2 \gamma(x)-\alpha)^{-2 n(x)-1} \leq \sum_{n \in \mathbf{Z}}\left(\left|B^{n} x\right|_{Q}^{2} \wedge 1\right) b^{-n \alpha} \\
\leq C_{7}\left(\alpha^{-1}+(2 \gamma(x)-\alpha)^{-2 n(x)-1}\right) \text { for } 0<\alpha<2 \gamma(x) .
\end{gathered}
$$


If $\alpha \geq 2 \gamma(x)$, then

$$
\sum_{n \in \mathbf{Z}}\left(\left|B^{n} x\right|_{Q}^{2} \wedge 1\right) b^{-n \alpha}=\infty
$$

Proof. Let $|x|_{Q}$ be defined by $|x|_{Q}=\int_{0}^{1}\left(\mid n Q_{x \mid / u}\right) d u$ also to $x \in \mathbf{C}^{d}$. We have

$$
\begin{aligned}
B^{k} T_{j} x & =\left(\beta_{j}+\left(B-\beta_{j}\right)\right)^{k} T_{j} x \\
& =\beta_{j}^{k} \sum_{l=0}^{n(x, j) \wedge k}\left(\begin{array}{l}
k \\
l
\end{array}\right) \beta_{j}^{-l}\left(B-\beta_{j}\right)^{l} T_{j} x .
\end{aligned}
$$

Thus

$$
\left|B^{k} x\right|_{Q} \leq C_{8} \beta(x)^{k} \sum_{j=1}^{p} k^{n(x, j)} \quad k \geq 1 .
$$

Hence we have (4.4). It follows from (4.8) that there are $b_{4}(x)$ and $b_{5}(x)$ such that, for $k \geq b_{4}(x)$,

$$
\begin{aligned}
\left|B^{k} T_{j} x\right|_{Q} & \geq 2^{-1}\left|\beta_{j}\right|^{k} k^{n(x, j)}(n(x, j) !)^{-1}\left|\left(B-\beta_{j}\right)^{n(x, j)} T_{j} x\right|_{Q} \\
& \geq b_{5}(x)\left|\beta_{j}\right|^{k} k^{n(x, j)}
\end{aligned}
$$

for all $j$ satisfying $T_{j} x \neq 0$. Choose a norm $\|\cdot\|$ in $\mathbf{C}^{d}$ as $\|x\|=\sum_{j=1}^{p}\left|T_{j} x\right|_{Q}$. Since arbitrary two norms are equivalent, we have $C_{9}|x|_{Q} \leq\|x\| \leq C_{10}|x|_{Q}$. Choosing $j$ such that $\beta(x)=\beta_{j}$ and $n(x)=n(x, j)$, we obtain

$$
\left|B^{k} x\right|_{Q} \geq C_{10}^{-1}\left\|B^{k} x\right\| \geq C_{10}^{-1}\left|B^{k} T_{j} x\right|_{Q} .
$$

Hence (4.5) follows. Let $0<\alpha<2 \gamma(x)$. Note that $\sum_{k=-\infty}^{0} b^{-k \alpha}=\left(1-b^{\alpha}\right)^{-1}$ and $C_{11} \alpha^{-1} \leq\left(1-b^{\alpha}\right)^{-1} \leq C_{12} \alpha^{-1}$. We see from (4.4) that

$$
\begin{aligned}
\sum_{k=1}^{\infty}\left|B^{k} x\right|_{Q}^{2} b^{-k \alpha} & \leq C_{5}^{2} \sum_{k=1}^{\infty} \beta(x)^{2 k} k^{2 n(x)} b^{-k \alpha} \\
& =C_{5}^{2} \sum_{k=1}^{\infty} b^{k(2 \gamma(x)-\alpha)} k^{2 n(x)} \leq C_{13}(2 \gamma(x)-\alpha)^{-2 n(x)-1} .
\end{aligned}
$$

This proves the second inequality in (4.6). The first inequality is obtained from (4.5) as follows. We have

$$
\begin{aligned}
\sum_{k \geq b_{1}(x)}\left|B^{k} x\right|^{2} b^{-k \alpha} & \geq b_{2}(x)^{2} \sum_{k \geq b_{1}(x)} b^{k(2 \gamma(x)-\alpha)} k^{2 n(x)} \\
& \geq b_{6}(x)(2 \gamma(x)-\alpha)^{-2 n(x)-1}
\end{aligned}
$$

for some $b_{6}(x)>0$. Hence the first inequality in (4.6) is obtained. The proof of (4.7) for $\alpha \geq 2 \gamma(x)$ is similar.

PROOF OF THEOREM 4.1. In the following the conditions (a)-(d) refer to those in Proposition 2.1. 
(i) Let $\left\{\nu_{0}, g_{n}, n \in \mathbf{Z}\right\}$ be the $S_{B}$-representation of $\nu$. Suppose that $\mu \in L_{\infty}(b, Q)$. It follows from Propositions 2.2 and 2.3 that, for $\nu_{0}$-a.e. $x$, there exists a measure $\Gamma_{x}$ such that

$$
g_{n}(x)=\int_{[0, \infty)} b^{n \alpha} \Gamma_{x}(d \alpha)
$$

By (c), $\Gamma_{x}$ is nonzero. By (d), for any $E \in \mathcal{B}\left(\mathbf{R}^{d}\right)$,

$$
\nu(E)=\int_{S_{B}} v_{0}(d x) \sum_{n \in \mathbf{Z}} \int_{[0, \infty)} 1_{E}\left(B^{-n} x\right) b^{n \alpha} \Gamma_{x}(d \alpha)
$$

Note that for any nonnegative measurable function $h(\alpha)$ on $[0, \infty), \int h(\alpha) \Gamma_{x}(d \alpha)$ is measurable in $x$. By (b),

$$
\infty>\sum_{n \geq 0} \int_{S_{B}} g_{n}(x) \nu_{0}(d x)=\int_{S_{B}} \nu_{0}(d x) \int_{[0, \infty)} \Gamma_{x}(d \alpha) \sum_{n \geq 0} b^{n \alpha} .
$$

When $\alpha=0, \sum_{n \geq 0} b^{n \alpha}=\infty$. Hence

$$
\int_{S_{B}} \nu_{0}(d x) \Gamma_{x}(\{0\})=0
$$

Next we have, by (d),

$$
\begin{aligned}
\infty & >\int_{\mathbf{R}^{d}}\left(|y|_{Q}^{2} \wedge 1\right) \nu(d y) \\
& =\sum_{n \in \mathbf{Z}} \int_{S_{B}}\left(\left|B^{-n} x\right|_{Q}^{2} \wedge 1\right) \nu_{0}(d x) \int_{(0, \infty)} b^{n \alpha} \Gamma_{x}(d \alpha) \\
& =\int_{S_{B}} \nu_{0}(d x) \int_{(0, \infty)} \Gamma_{x}(d \alpha) \sum_{n \in \mathbf{Z}} b^{n \alpha}\left(\left|B^{-n} x\right|_{Q}^{2} \wedge 1\right) .
\end{aligned}
$$

Thus by Lemma 4.1, we have

$$
\int_{S_{B}} \nu_{0}(d x) \int_{[2 \gamma(x), \infty)} \Gamma_{x}(d \alpha)=0
$$

concluding (4.1), and the integrability condition (4.2) is also proved.

Set

Conversely suppose that $v$ has the representation (4.1) with $v_{0}$ and $\Gamma_{x}$ satisfying (4.2).

$$
g_{n}(x)=\int_{(0,2 \gamma(x))} b^{n \alpha} \Gamma_{x}(d \alpha), \quad n \in \mathbf{Z}
$$


Then (4.1) and (4.9) imply (d). We observe that $g_{n}(x)$ in (4.9) satisfies (a), (b), and (c). As to (b),

$$
\begin{aligned}
\int_{S_{B}} \nu_{0}(d x) & \sum_{n \in \mathbf{Z}}\left(\left|B^{-n} x\right|_{Q}^{2} \wedge 1\right) g_{n}(x) \\
& =\int_{S_{B}} v_{0}(d x) \sum_{n \in \mathbf{Z}}\left(\left|B^{-n} x\right|_{Q}^{2} \wedge 1\right) \int_{(0,2 \gamma(x))} b^{n \alpha} \Gamma_{x}(d \alpha) \\
& =\int_{S_{B}} v_{0}(d x) \int_{(0,2 \gamma(x))} \Gamma_{x}(d \alpha) \sum_{n \in \mathbf{Z}} b^{n \alpha}\left(\left|B^{-n} x\right|_{Q}^{2} \wedge 1\right)<\infty
\end{aligned}
$$

by (4.2). (a) and (c) are obvious because $\Gamma_{x}(d \alpha)$ is nonzero for each $x$. Therefore $\left\{v_{0}, g_{n}, n \in\right.$ $Z$ \} is the $S_{B}$-representation of $v$. It follows from (4.9) and Proposition 2.2 that $g_{n}(x)$ is completely monotone in $n \in \mathbf{Z}$. Thus, by Proposition 2.3, $\mu \in L_{\infty}(b, Q)$.

(ii) In order to see that $v$ is the Lévy measure of some $\mu$, it is enough to show that $v(\{0\})=0$ and that $\int_{\mathbf{R}^{d}}\left(|x|^{2} \wedge 1\right) v(d x)<\infty$. The former is obvious from (4.1). The latter follows from (4.2) since $|x| \leq$ const. $\times|x|_{Q}$.

(iii) To show the uniqueness, suppose that both $\left\{v_{0}, \Gamma_{x}\right\}$ and $\left\{\tilde{v}_{0}, \tilde{\Gamma}_{x}\right\}$ represent $v$. Let

$$
g_{n}(x)=\int_{(0,2 \gamma(x))} b^{n \alpha} \Gamma_{x}(d \alpha), \quad \tilde{g}_{n}(x)=\int_{(0,2 \gamma(x))} b^{n \alpha} \tilde{\Gamma}_{x}(d \alpha) .
$$

By the proof above, $\left\{\nu_{0}, g_{n}\right\}$ and $\left\{\tilde{\nu}_{0}, \tilde{g}_{n}\right\}$ are $S_{B}$-representations of $\nu$. Thus by the uniqueness of them in Proposition 2.1, there exists a Borel measurable function $h(x)$ with $0<h(x)<\infty$ such that $\tilde{\nu}_{0}(d x)=h(x) \nu_{0}(d x)$ and $g_{n}(x)=h(x) \tilde{g}_{n}(x)$, $\nu_{0}$-a.e. $x$ for any $n \in \mathbf{Z}$. Thus by the uniqueness assertion in Proposition 2.2(i)(b) and by (4.10), we conclude that $\Gamma_{x}(d \alpha)=$ $h(x) \tilde{\Gamma}_{x}(d \alpha)$. The assertion (4.3) for $\Gamma_{x}$ follows from (4.2) and (4.6).

\section{Proof of Theorem 1.1.}

We first show that $O S S(b, Q) \subset L_{\infty}(b, Q)$. Let $\mu \in O S S(b, Q)$. That is, for some $0<a<1$ and $c \in \mathbf{R}^{d}$,

$$
\hat{\mu}(z)^{a}=\hat{\mu}\left(b^{Q^{\prime}} z\right) e^{i\langle c, z\rangle} .
$$

Then

$$
\hat{\mu}(z)=\hat{\mu}\left(b^{Q^{\prime}} z\right) \hat{\rho}(z)
$$

with

$$
\hat{\rho}(z)=\hat{\mu}(z)^{1-a} e^{i\langle c, z\rangle} .
$$

To show that $\mu \in L_{\infty}(b, Q)$, by the definition, it is enough to show that $\rho \in L_{\infty}(b, Q)$. Since $\mu \in I\left(\mathbf{R}^{d}\right), \rho \in I\left(\mathbf{R}^{d}\right)$. Hence by (5.2), $\mu \in L_{0}(b, Q)$. Since $L_{0}(b, Q)$ is $Q$-completely closed in the strong sense as mentioned in Section $1, \rho$ in (5.3) is in $L_{0}(b, Q)$. Thus by the definition, (5.2) implies that $\mu \in L_{1}(b, Q)$. Repeating this argument, we conclude that 
$\mu \in L_{m}(b, Q)$ for any $1 \leq m<\infty$ and therefore $\mu \in L_{\infty}(b, Q)$. Hence $\operatorname{OSS}(b, Q) \subset$ $L_{\infty}(b, Q)$.

Since $L_{\infty}(b, Q)$ is $Q$-completely closed in the strong sense, it only remains to prove "the smallest". Let $K$ be any $Q$-completely closed class in the strong sense containing $\operatorname{OS} S(b, Q)$. First, notice the following fact. Let $\alpha>0$ and $r(z, x)=e^{i\langle z, x\rangle}-1-i\langle z, x\rangle /\left(1+|x|^{2}\right)$. If $\nu_{0}$ is a finite measure concentrated on $S_{B} \cap\{x: 2 \gamma(x)>\alpha\}$ satisfying

$$
\int_{S_{B}} \nu_{0}(d x) \sum_{n<0} b^{n \alpha}\left|b^{-n Q} x\right|^{2}<\infty
$$

and if $\mu$ is a distribution with

$$
\hat{\mu}(z)=\exp \left\{\int_{S_{B}} \nu_{0}(d x) \sum_{n \in \mathbf{Z}} b^{n \alpha} r\left(z, b^{-n Q} x\right)\right\},
$$

then $\mu \in O S S(b, Q)$, since (5.1) holds with $a=b^{\alpha}$. Now let $\mu$ be a purely non-Gaussian distribution in $L_{\infty}(b, Q)$. Then its Lévy measure is represented as in Theorem 4.1, and we have

$$
\hat{\mu}(z)=\exp \left\{i\langle\gamma, z\rangle+\int_{S_{B}} \nu_{0}(d x) \int_{(0,2 \gamma(x))} \Gamma(d \alpha) \sum_{n \in \mathbf{Z}} b^{n \alpha} r\left(z, b^{\left.-n Q_{x}\right)}\right\}\right.
$$

with some $\gamma \in \mathbf{R}^{d}$. For $\varepsilon>0$, define $\mu_{\varepsilon}$ by

$$
\hat{\mu}_{\varepsilon}(z)=\exp \left\{i\langle\gamma, z\rangle+\int_{S_{B}} \nu_{0}(d x) \int_{(\varepsilon, 2 \gamma(x)-\varepsilon)} \Gamma_{x}(d \alpha) \sum_{n \in \mathbf{Z}} b^{n \alpha} r\left(z, b^{\left.-n Q_{x}\right)}\right\} .\right.
$$

Since the number of the possible values of $\gamma(x)$ is finite, $2 \gamma(x)-\varepsilon>\varepsilon$ for all $x$, if $\varepsilon$ is small enough. Then, for fixed $\varepsilon>0$, we can choose measures $\Gamma_{x}^{(n)}(d \alpha)$ satisfying the following conditions: $\Gamma_{x}^{(n)}(d \alpha)$ is concentrated on the points $\left\{k 2^{-n}, k=1,2, \cdots\right\} \cap(\varepsilon, 2 \gamma(x)-\varepsilon)$, $\Gamma_{x}^{(n)}(d \alpha)$ converges to $\Gamma_{x}(d \alpha)$ for each $x \in S_{B}$ as $n \rightarrow \infty$, the total mass of $\Gamma_{x}^{(n)}(d \alpha)$ does not exceed that of $\Gamma_{x}(d \alpha)$ for each $x \in S_{B}$, and $\left\{\Gamma_{x}^{(n)}\right\}$ is measurable in $x$. Define $\mu_{\varepsilon}^{(n)}$ by

$$
\hat{\mu}_{\varepsilon}^{(n)}(z)=\exp \left\{i\langle\gamma, z\rangle+\int_{S_{B}} \nu_{0}(d x) \int_{(\varepsilon, 2 \gamma(x)-\varepsilon)} \Gamma_{x}^{(n)}(d \alpha) \sum_{j \in \mathbf{Z}} b^{j \alpha} r\left(z, b^{\left.-j Q_{x}\right)}\right\} .\right.
$$

We see that $\mu_{\varepsilon}^{(n)}$ is a convolution of a finite number of purely non-Gaussian distributions in $O S S(b, Q)$. Hence $\mu_{\varepsilon}^{(n)} \in K$. We see from (4.6) that, for any fixed $\varepsilon>0$,

$$
C_{14} \leq \sum_{n \in \mathbf{Z}} b^{n \alpha}\left(\left|b^{-n Q} x\right|_{Q}^{2} \wedge 1\right) \leq C_{15} \quad \text { for } \alpha \in(\varepsilon, 2 \gamma(x)-\varepsilon) \text { and } x \in S_{B} .
$$

Hence, by (4.2),

$$
\int_{S_{B}} v_{0}(d x) \Gamma_{x}((\varepsilon, 2 \gamma(x)-\varepsilon))<\infty .
$$

We show that, for fixed $z \in \mathbf{R}^{d}, \sum_{n \in \mathbf{Z}} b^{n \alpha} r\left(z, b^{\left.-n Q_{x}\right)}\right.$ is bounded in $\alpha \in(\varepsilon, 2 \gamma(x)-\varepsilon)$ and $x \in S_{B}$, and continuous in $\alpha$. Since $|r(z, x)| \leq C_{16}\left(|x|_{Q}^{2} \wedge 1\right)$, we see the boundedness from (5.4). The continuity is obvious. Thus by (5.5) and Lebesgue's dominated convergence 
theorem, we have that as $n \rightarrow \infty \hat{\mu}_{\varepsilon}^{(n)} \rightarrow \hat{\mu}_{\varepsilon}(z)$, and that $\mu_{\varepsilon} \in K$. Finally, $\hat{\mu}_{\varepsilon} \rightarrow \hat{\mu}$ as $\varepsilon \downarrow 0$. Thus $\mu \in K$. This proves that if $\mu \in L_{\infty}(b, Q)$ is purely non-Gaussian, then $\mu \in K$.

If $\mu \in L_{\infty}(b, Q)$ is Gaussian, then by Theorem $3.4, \mu$ is a convolution of finite number of Gaussian distributions in $O S S(b, Q) \subset K$, and thus $\mu \in K$. As Proposition 2.3 shows, any $\mu \in L_{\infty}(b, Q)$ is decomposable in $L_{\infty}(b, Q)$ as the convolution of a Gaussian and a purely non-Gaussian. Hence $L_{\infty}(b, Q) \subset K$ and the proof is complete.

\section{Remarks on the case $Q=I$.}

Let us consider the case $Q=I$. Let $0<b<1$. The classes $L_{m}(b, I), 0 \leq m \leq$ $\infty$, were introduced by Maejima and Naito [MN98], of which the paper [MSW99] was a matrix generalization on $\mathbf{R}^{d}$. Distributions in $L_{0}(b, I)$ are called semi-selfdecomposable. Distributions in $L_{\infty}(b, I)$ should be called completely semi-selfdecomposable. The class $O S S(b, I)$ consists of $\mu \in \mathcal{P}\left(\mathbf{R}^{d}\right)$ that satisfies $\hat{\mu}(z)^{a}=\hat{\mu}(b z) e^{i\langle c, z\rangle}$ for some $0<a<1$ and $c \in \mathbf{R}^{d}$; namely it is the class of $\mu \in \mathcal{P}\left(\mathbf{R}^{d}\right)$ that satisfies $\hat{\mu}(z)^{b^{\alpha}}=\hat{\mu}(b z) e^{i\langle c, z\rangle}$ for some $0<\alpha \leq 2$ and $c \in \mathbf{R}^{d}$. Thus distributions in $O S S(b, I)$ are exactly semi-stable distributions studied by many authors beginning with Lévy [L37]. Now we have $B=b I,|x|_{Q}=|x|$, and $S_{B}=\left\{x \in \mathbf{R}^{d}: b<|x| \leq 1\right\}$. We write $S_{B}$ as $S_{b}$. Further we have $p=q=1$, $b_{1}=b, n_{1}=1$, and $f(\zeta)=\zeta-b$. Since $\Psi_{B}(A)=\left(1-b^{2}\right) A$, Proposition 2.3 shows that all Gaussian distributions are in $L_{\infty}(b, I)$. Since $\beta(x)=b$ and $\gamma(x)=1$ for all $x \in \mathbf{R}^{d}$, the following result is obtained from Theorem 4.1.

THEOREM 6.1. (i) Suppose that $\mu$ is in $I\left(\mathbf{R}^{d}\right)$ with nonzero Lévy measure $v$. Then $\mu \in L_{\infty}(b, I)$ if and only if $v$ is expresed as

$$
v(E)=\int_{S_{b}} \nu_{0}(d x) \int_{(0,2)} \Gamma_{x}(d \alpha) \sum_{n \in \mathbf{Z}} b^{n \alpha} 1_{E}\left(b^{-n} x\right), \quad E \in \mathcal{B}\left(\mathbf{R}^{d}\right)
$$

where $v_{0}$ is a nonzero finite measure on $S_{b}$ and $\Gamma_{x}, x \in S_{b}$, are measures on $(0,2)$ measurable in $x$ satisfying

$$
\int_{(0,2)}\left(\frac{1}{\alpha}+\frac{1}{2-\alpha}\right) \Gamma_{x}(d \alpha)=1
$$

(ii) If a nonzero finite measure $v_{0}$ on $S_{b}$ and measures $\Gamma_{x}, x \in S_{b}$, on $(0,2)$ measurable in $x$ satisfying (6.2) are given, then the measure $v$ defined by (6.1) is the Lévy measure of some $\mu \in I\left(\mathbf{R}^{d}\right)$.

(iii) If $\mu \in L_{\infty}(b, I)$ with nonzero Lévy measure $v$, then the measure $v_{0}$ in (i) is uniquely determined by $v$ and the measures $\Gamma_{x}, x \in S_{b}$, are unique in the sense that $\tilde{\Gamma}_{x}=\Gamma_{x}$ for $v_{0}$-a.e. $x$ for any $\tilde{\Gamma}_{x}$ which expresses $v$ by (6.1) in place of $\Gamma_{x}$.

Proof. (i) We apply Theorem 4.1. Note that we do not assume that $\mu$ is purely nonGaussian, since all Gaussians are in $L_{\infty}(b, I)$. Suppose that $\mu \in L_{\infty}(b, I)$. Then we get 
(6.1) with some $\nu_{0}$ and $\Gamma_{x}$ satisfying

$$
\int_{S_{b}} \nu_{0}(d x) \int_{(0,2)} \Gamma_{x}(d \alpha) \sum_{n \in \mathbf{Z}} b^{n \alpha}\left(\left(b^{-2 n}|x|^{2}\right) \wedge 1\right)<\infty .
$$

This is equivalent to

$$
\int_{S_{b}} \nu_{0}(d x) \int_{(0,2)} \Gamma_{x}(d \alpha)\left(\sum_{n \geq 0} b^{n \alpha}+\sum_{n<0} b^{-n(2-\alpha)}\right)<\infty .
$$

Since $\sum_{n \geq 0} b^{n \alpha} \sim C_{17} / \alpha$ as $\alpha \downarrow 0$ and $\sum_{n<0} b^{-n(2-\alpha)} \sim C_{18} /(2-\alpha)$ as $\alpha \uparrow 2$, the condition is equivalent to

$$
\int_{S_{B}} \nu_{0}(d x) \int_{(0,2)}\left(\frac{1}{\alpha}+\frac{1}{2-\alpha}\right) \Gamma_{x}(d \alpha)<\infty
$$

Let $h(x)=\int_{(0,2)}(1 / \alpha+1 /(2-\alpha)) \Gamma_{x}(d \alpha)$ and use $h(x) \nu_{0}(d x)$ and $(1 / h(x)) \Gamma_{x}(d \alpha)$ in place of $\nu_{0}$ and $\Gamma_{x}$ to obtain (6.2). The converse is similarly proved. (ii) and (iii) follow from Theorem 4.1(ii) and (iii), respectively.

Another form of the theorem above is as follows.

THEOREM 6.2. (i) Suppose that $\mu \in I\left(\mathbf{R}^{d}\right)$ with nonzero Lévy measure $v$. Then $\mu \in L_{\infty}(b, I)$ if and only if

$$
\nu(E)=\int_{(0,2)} \Gamma(d \alpha) \sum_{n \in \mathbf{Z}} b^{n \alpha} v_{\alpha}\left(\left(b^{n} E\right) \cap S_{b}\right), \quad E \in \mathcal{B}\left(\mathbf{R}^{d}\right),
$$

where $\Gamma$ is a nonzero measure on $(0,2)$ with

$$
\int_{(0,2)}\left(\frac{1}{\alpha}+\frac{1}{2-\alpha}\right) \Gamma(d \alpha)<\infty
$$

and $v_{\alpha}, \alpha \in(0,2)$, are probability measures on $S_{b}$, measurable in $\alpha$.

(ii) If a nonzero measure $\Gamma$ on $(0,2)$ satisfying $(6.4)$ and probability measures $\nu_{\alpha}$, $\alpha \in(0,2)$, on $S_{b}$ measurable in $\alpha$ are given, then the measure $v$ defined by $(6.3)$ is the Lévy measure of some $\mu \in I\left(\mathbf{R}^{d}\right)$.

(iii) If $\mu \in L_{\infty}(b, I)$ with nonzero Lévy measure $\nu$, then the measure $\Gamma$ in (i) is uniquely determined by $v$ and the probability measures $v_{\alpha}, \alpha \in(0,2)$, on $S_{b}$ are unique in the sense that $\tilde{v}_{\alpha}=v_{\alpha}$ for $\Gamma$-a.e. $\alpha$ for any $\tilde{v}_{\alpha}$ that expresses $v$ by (6.3) in place of $v_{\alpha}$.

PROOF: In order to go to the representation (6.3) from (6.1), consider the probability measure $\left(1 / \nu_{0}\left(S_{b}\right)\right) \nu_{0}(d x)((1 / \alpha)+1 /(2-\alpha)) \Gamma_{x}(d \alpha)$ on $S_{b} \times(0,2)$ and apply the existence theorem for conditional distributions. Transfer in the reverse direction is similar.

Finiteness and infiniteness of the moments of distributions in $L_{\infty}(b, I)$ are determined only by the measure $\Gamma$. This is an application of Theorem 6.2 .

THEOREM 6.3. Let $\mu$ be a distribution in $L_{\infty}(b, I)$ with nonzero Lévy measure $v$. Let $\Gamma(d \alpha)$ be the nonzero measure on $(0,2)$ uniquely determined by $v$ in Theorem 6.2 . Let 
$\alpha_{0} \in[0,2)$ be the infimum of the support of $\Gamma$. Then, finiteness and infiniteness of $M_{\eta}=$ $\int_{\mathbf{R}^{d}}|x|^{\eta} \mu(d x)$ are as follows.

(i) If $\eta>\alpha_{0}$, then $M_{\eta}=\infty$.

(ii) If $\alpha>0$ and $0<\eta<\alpha_{0}$, then $M_{\eta}<\infty$.

(iii) If $\alpha>0$ and $\Gamma\left(\left\{\alpha_{0}\right\}\right)>0$, then $M_{\alpha_{0}}=\infty$.

(iv) If $\alpha_{0}>0$ and $\Gamma\left(\left\{\alpha_{0}\right\}\right)=0$, then $M_{\alpha_{0}}$ is finite or infinite according as $\int_{\left(\alpha_{0}, 2\right)}$ $\left(1 /\left(\alpha-\alpha_{0}\right)\right) \Gamma(d \alpha)$ is finite or infinite.

PROOF. It is known that $M_{\eta}<\infty$ if and only if $\int_{|x|>1}|x|^{\eta} v(d x)<\infty$ (Kruglov [K70]). We have, from (6.3),

$$
\begin{aligned}
\int_{|x|>1}|x|^{\eta} v(d x) & =\int_{(0,2)} \Gamma(d \alpha) \int_{S_{b}} v_{\alpha}(d x) \sum_{n \in \mathbf{Z}} b^{n \alpha}\left|b^{-n} x\right|^{\eta} 1_{E}\left(b^{-n} x\right) \\
& =\int_{(0,2)} \Gamma(d \alpha) \int_{S_{b}} \nu_{\alpha}(d x)|x|^{\eta} \sum_{n \geq 1} b^{n(\alpha-\eta)},
\end{aligned}
$$

where $E=\{x:|x|>1\}$. If $\eta>\alpha_{0}$, then $\sum_{n \geq 1} b^{n(\alpha-\eta)}=\infty$ for $\alpha \in\left[\alpha_{0}, \eta\right)$ and $\int_{|x|>1}|x|^{\eta} v(d x)=\infty$. If $\alpha_{0}>0$ and $0<\eta<\alpha_{0}$, then

$$
\int_{|x|>1}|x|^{\eta} v(d x) \leq \frac{1}{1-b^{\alpha_{0}-\eta}} \int_{\left[\alpha_{0}, 2\right)} \Gamma(d \alpha) \int_{S_{b}}|x|^{\eta} v_{\alpha}(d x)<\infty .
$$

If $\alpha_{0}>0$ and $\Gamma\left(\left\{\alpha_{0}\right\}\right)>0$, then

$$
\int_{|x|>1}|x|^{\alpha_{0}} v(d x) \geq \Gamma\left(\left\{\alpha_{0}\right\}\right) \int_{S_{b}} v_{\alpha_{0}}(d x)|x|^{\alpha_{0}} \sum_{n \geq 1} 1=\infty .
$$

Hence we obtain (i), (ii), and (iii). Consider the final case, $\alpha_{0}>0$ and $\Gamma\left(\left\{\alpha_{0}\right\}\right)=0$. Since $\sum_{n \geq 1} b^{n\left(\alpha-\alpha_{0}\right)} \sim C_{19} /\left(\alpha-\alpha_{0}\right)$ as $\alpha \downarrow \alpha_{0}$, we have

$$
C_{20} \int_{\left(\alpha_{0}, 2\right)} \frac{1}{\alpha-\alpha_{0}} \Gamma(d \alpha) \leq \int_{|x|>1}|x|^{\alpha_{0}} v(d x) \leq C_{21} \int_{\left(\alpha_{0}, 2\right)} \frac{1}{\alpha-\alpha_{0}} \Gamma(d \alpha) .
$$

Hence the assertion (iv) follows.

\section{References}

[J77] R. JAJTE, Semi-stable probability measures on $\mathbf{R}^{N}$, Studia Math. 61 (1977), 29-39.

[J83] Z. J. JUREK, Limit distributions and one-parameter groups of linear operators on Banach spaces. J. Multivariate Anal. 13 (1983), 578-604.

[JM93] Z. J. JUREK and J. D. MASON, Operator-Limit Distributions in Probability Theory, John Wiley (1993).

[K80] W. KRAKOWIAK, Operator semi-stable probability measures on Banach spaces, Colloq. Math. 43 (1980), 351-363.

[K70] V. M. Kruglov, A note on infinitely divisible distributions, Theory Probab. Appl. 15 (1970), 319-324.

[LR80] R. G. LAHA and V. K. ROHATGI, Operator semi-stable probability measures on Hilbert space, Bull. Austral. Math. Soc. 22 (1980), 397-406. (Corrigenda, 22, 479-480.)

[L37] P. LÉVY, Théorie de l'addition des variables aléatoires, Gauthier-Villars (1937).

[Ł81] A. ŁUCZAK, Operator semi-stable probability measures on $\mathbf{R}^{N}$, Colloq. Math. 45 (1981), 287-300. (Corrigenda, 52 (1987), 167-169.) 
[Ł91] A. ŁUCZAK, Operator-semi-stable operator Lévy’s measures on finite dimensional vector spaces, Probab. Theory Related Fields 90 (1991), 317-340.

[MN98] M. MAEJIMA and Y. NAITO, Semi-selfdecomposable distributions and a new class of limit theorems, Probab. Theory Related Fields 112 (1998), 13-31.

[MSW99] M. MaEJima, K. SATo and T. WATANABE, Operator semi-selfdecomposability, $(C, Q)$-decomposability and related nested classes, Tokyo J. Math. 22 (1999), 473-509.

[SY85] K. SATO and M. YAMAZATO, Completely operator-selfdecomposable distributions and operator-stable distributions, Nagoya Math. J. 97 (1985), 71-94.

Present Addresses:

MAKoto MAEJIMA

DEPARTMENT OF MATHEMATICS, KeIO UNIVERSITY, HIYOSHI, YOKOHAMA, 223-8522 JAPAN.

e-mail: maejima@math.keio.ac.jp

KEN-ITI SATO

HACHIMAN-YAMA 1101-5-103, TENPAKU-KU, NAGOYA, 468-0074 JAPAN.

e-mail: ken-iti.sato@nifty.ne.jp

TOSHIRo WATANABE

CENTER For Mathematical Sciences, The University of Aizu, AizU-WAKamatsu, FUKUSHIMA, 965-8580 JAPAN.

e-mail: t-watanb@u-aizu.ac.jp 